\title{
评 述祝贺过增元院士85华诞专辑
}

\section{场协同原理: 它的科学之美及与积的关系}

\author{
陶文铨 ${ }^{1,2 *}$, 何雅玲 ${ }^{1,2}$
}

1 西安交通大学热流科学与工程教育部重点实验室, 西安 710049;

2 西安交通大学热科学与工程国际合作联合实验室(筹), 西安 710049

*E-mail:wqtao@mail.xjtu.edu.cn

收稿日期: 2021-04-01; 接受日期: 2021-05-28; 网络版发表日期: 2021-09-02

国家重点基础研究发展计划(973计划)(编号: 2013CB228304)和国家自然科学基金重大项目(批准号: 52090064)资助

摘要 本文从科学原理之美的视角, 从6个方面阐述了强化对流传热的场协同原理之美, 论述了其概念清晰、推 导优雅、应用广泛，且具有自然性、对称性和比拟性; 最后揭示了场协同原理与筫理论之间的内在联系，阐明了 场协同原理与筫理论相互论证、相辅相成, 对传热学的发展具有重要完善和推动作用.

关键词 科学之美, 场协同原理, 椥理论

\section{1 引言}

“美”本是一种心理学或社会学的概念. 我国著名 的美学家朱光潜先生说过:“持实用的态度看事物，它 们都只是实际生活的工具或障碍物，都只能引起欲念 或嫌恶. 要见出事物本身的美, 我们一定要从实用世 界跳开，以“无所为而为’的精神欣赏它们本身的形象. 总而言之, 美和实际人生有一个距离, 要见出事物本 身的美, 须把它摆在适当的距离之外去看. ${ }^{[}{ }^{[1]}$

本文中, 我们将讨论科学原理之美. “美”是许多数 学家和物理学家追求的目标, 他们喜欢优美(或漂亮) 的结果、优美的公式、优美的方法、优美的证明、优 美的理论、优美的插图等.

数学的美表现在她的简约之美、对称之美、功能 强大之美、应用普遍性之美 ${ }^{[2]}$. 我国著名数学家华罗 庚先生说过:“就数学本身而言，是壮丽多彩、千姿百
态、引人入胜的……论为数学枯燥乏味只是看到了数 学的严谨性, 而没有体会出数学的内在美! ’ ${ }^{[3]}$ 例如, 彭 实戈先生认为非线性期望理论的发展体现了数学之精 妙 $^{[4]}$, 其在信息科学、经济学等领域受到了广泛的应 用. 又例如, 在数学界有研究者把以下的欧拉公式评 价为最美的公式 ${ }^{[5]}$ :

$\mathrm{e}^{\pi \mathrm{i}}+1=0$,

其中 $\mathrm{e}$ 为自然对数的底, $\pi$ 为圆周率, $i$ 为虚数的 单位 $\sqrt{-1}$.

那么什么是科学原理之美呢? 或者说, 科学原理 之美表现在哪些方面呢? 综合文献[6,7]中的观点, 科 学之美可以表现在以下 6 个方面: (1) 概念清晰(clear basic concept); (2) 推导优雅(elegant in derivation); (3) 应用广泛(high unification); (4) 具有自然性(naturarity); （5）具有对称性(symmetry); (6) 具有类比性(analogy).

引用格式: 陶文铨, 何雅玲. 场协同原理: 它的科学之美及与积的关系. 中国科学: 技术科学, 2021, 51: 1155-1165 Tao W Q, He Y L. Field synergy principle: Its scientific beauty and relationship with entransy theory (in Chinese). Sci Sin Tech, 2021, 51: 1155-1165, doi: 10.1360/SST-2021-0138 
本文试图根据作者的理解从这 6 个方面来研讨场协同 原理的科学之美.

\section{2 场协同原理的科学之美}

\section{1 概念清晰}

1998年过增元先生及其同事对沿着图1所示的平 板流动的边界层流动的能量方程式(2)做了垂直于流 动方向的积分 ${ }^{[8]}$, 得到式(3).

$\rho c_{p}\left(u \frac{\partial T}{\partial x}+v \frac{\partial T}{\partial y}\right)=\frac{\partial}{\partial y}\left(k \frac{\partial T}{\partial y}\right)$,

其中, $\rho$ 为流体的密度, $c_{p}$ 为流体的定压比热容, $u$ 和 $v$ 分 别为流体在 $x$ 和 $y$ 方向上的速度, $T$ 为流体温度, $k$ 为流体 的导热系数.

$\int_{0}^{\delta_{\mathrm{t}}} \rho c_{p}\left(u \frac{\partial T}{\partial x}+v \frac{\partial T}{\partial y}\right) \mathrm{d} y=-\left.k \frac{\partial T}{\partial y}\right|_{\mathrm{w}}=q_{\mathrm{w}}$,

其中, $\delta_{\mathrm{t}}$ 为边界层厚度, $q_{\mathrm{w}}$ 是流体与壁面之间交换的热 流密度, 即单位面积上的对流传热量.

非常重要的是，作者将式(3)积分号中的对流项表 示成流体速度与流体温度梯度的点积:

$u \frac{\partial T}{\partial x}+v \frac{\partial T}{\partial y}=\mathbf{U} \cdot \nabla T=|\mathbf{U}||\nabla T| \cos \theta$,

其中, $\mathbf{U}$ 和 $\nabla T$ 分别为流体速度和温度梯度的向量表 达, $\theta$ 为流体速度和温度梯度的夹角.

显然, 速度与温度梯度的夹角越小 (或越接近 $180^{\circ}$ ), 则矢量点积的绝对值就越大. 根据美国英语的Webster 字典 ${ }^{[9]}$ : 当几个作用或力互相合作、联合时的情形称 为协同(synergy). 在这里两个作用就是速度与温度梯 度，当它们协同时两者的夹角小、点积就大，对流传 热就强烈(流体被加热时夹角应尽可能接近 $0^{\circ}$; 流体被

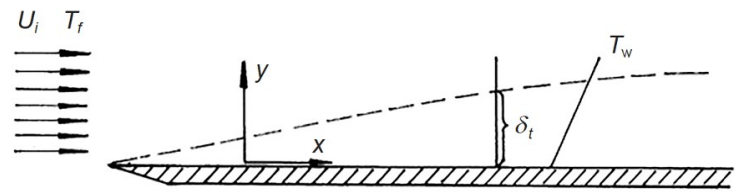

图 1 平板边界层流动

Figure 1 Flat boundary layer flow.

冷却时夹角应尽可能接近 $180^{\circ}$ ). 所以强化对流传热的 基本途径是改善速度与温度梯度之间的协同性，这就 是场协同原理(field synergy principle, FSP)的基本思 想. 上述分析虽然是从层流边界层型流动推导得出的, 对于椭圆型流动的对流传热及湍流传热也同样成立, 参见文献[10 12]的讨论.

上述分析简单、清晰, 只要是学习过传热学的大 学本科学生都能理解和掌握. 因此这一内容早在2006 年就写进了本科生的传热学教材, 参见文献[13].

从场协同原理可以得出对流传热的两种极端情 况, 如图2所示. 其中图2(a)所示情形是速度与温度梯 度垂直, 所以流体中只发生导热, 无论流速多大都对 传热强化毫无收益, 这是最差的情形; 在图2(b)中, 温 度梯度与速度同向(流体被加热)或反向(流体被冷却), 这是最理想的对流传热, 这时对流传热与流体速度成 正比.

值得指出, 场协同原理已经得到许多实验与数值 模拟的证实. 这里特别要提到赵天寿教授关于多孔介 质的传热实验 ${ }^{[14]}$ 及作者团队的实验 ${ }^{[15]}$.

文献[14]通过对图3(a)所示情形的理论分析得出

$N u=R e \cdot P r=P e$.

图3(b)所示结果表明, 在贝克莱数 $P e$ 小于 7 时理论 分析与实验结果一致. 这就是图2(b)中流体被加热的 情形, 即温度梯度与速度同向时，对流传热与流体速
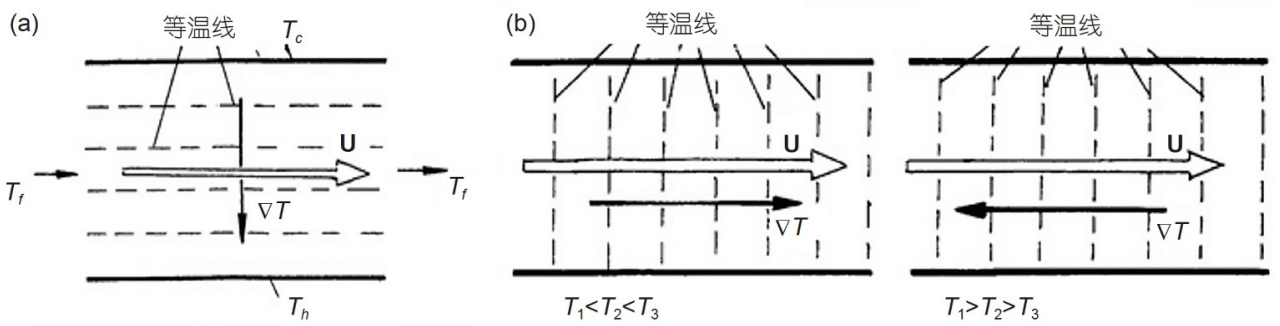

图 2 对流传热的两种极端情形. (a) 速度与温度梯度相互垂直; (b) 速度与温度梯度同向或反向

Figure 2 Two extreme cases of convective heat transfer. (a) Velocity is perpendicular to temperature gradient; (b) velocity is in the same or opposite direction to temperature gradient. 

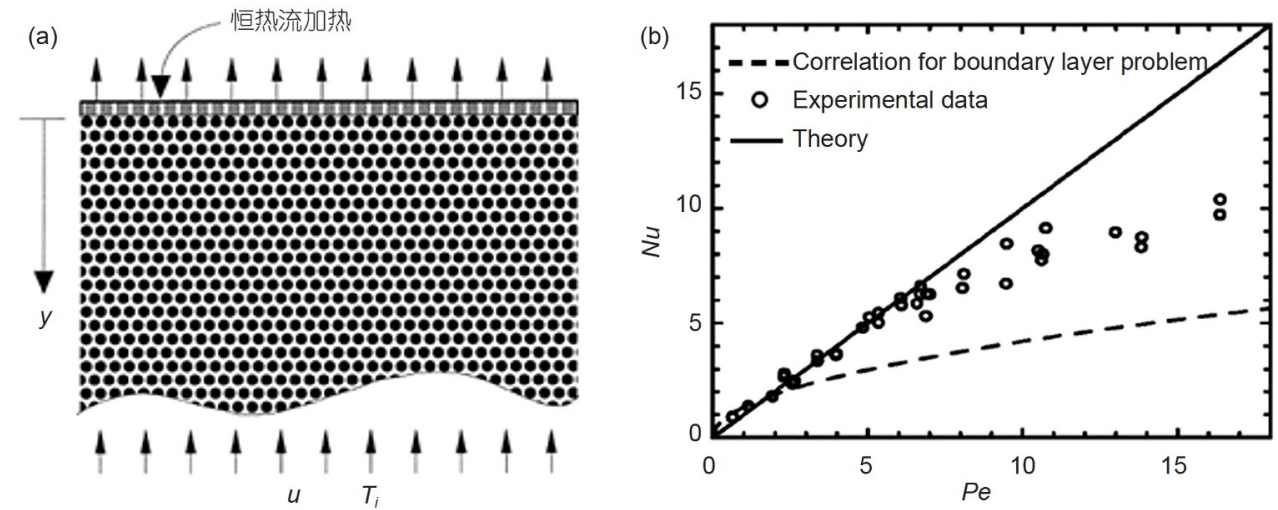

图 3 文献[14]的结果. (a) 流体流经一侧恒热流加热的多孔介质; (b) $N u$ 与 $P e$ 的关系

Figure 3 Results of ref. [14]. (a) The fluid flows through a porous medium heated by a constant heat flow on one side; (b) relationship between $N u$ and $P e$.

度成正比，而流速大于一定数值后由于流体的扰动会 使得等温线分布偏离温度梯度与流速完全同向的条 件，因此传热努谢尔数 $N u$ 不再与贝克莱数成正比. 文 献[14]的实验结果完美论证了场协同原理在温度梯度 与速度同向时的推论.

文献[15]设计了一个特殊的正方形传热通道，其 截面如图4所示. 试验时使得两侧热水与冷水的温度 变化各自维持在 $1^{\circ} \mathrm{C}$ 左右, 就近似地构建了两等温面 之间的传热, 其中空气的温度梯度从左侧指向到右侧, 而管道轴向的主流速度则与其垂直. 试验结果表明, 通 道冷热表面之间的传热量只与其温差有关，而与通道 中的流速无关(在实验数据的误差范围内), 如图 5 所示. 这就是图2(a)温度梯度与速度相互垂直的情形，即温

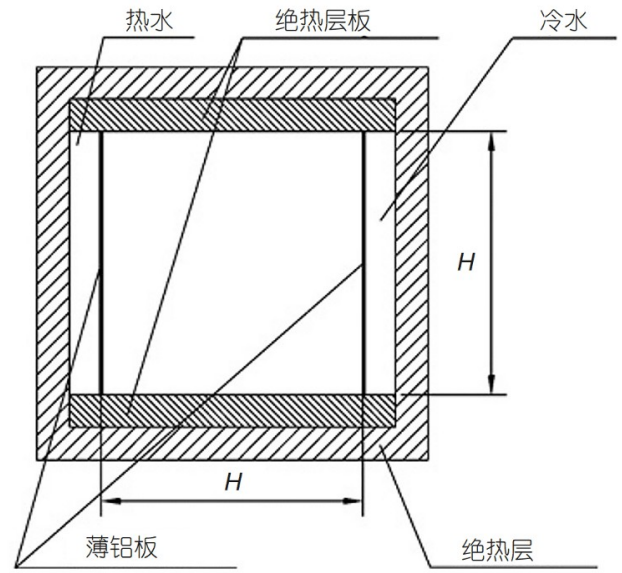

图 4 通道截面图

Figure 4 Channel cross section.
度梯度与速度垂直时，流体中只发生导热，流动对传 热强化无贡献. 文献[15]的实验结果完美论证了场协 同原理在温度梯度与速度垂直时的推论.

\section{2 推导优雅}

优雅者, 雅致、简单、清新也. 在场协同原理的推 导过程中只用了两步数学工具, 就是对边界层能量方 程做积分，以及把对流项表示成矢量点积的形式，没 有复杂的推理; 但这两步推导十分重要: 第1步把能量 方程直接和壁面的对流传热量联系了起来, 第2步中引 出了速度与温度梯度之间的夹角. 历史上1904年普朗 特提出了流动边界层理论，1921年坡尔豪森第一个求 解流体外掠平板边界层能量方程 ${ }^{[16]}$, 所以边界层能量 方程已经建立了半个多世纪，但这个速度与温度梯度 的夹角却一直隐含在对流项中，没有人发现其重要性; 一旦在经积分后的能量方程中把对流项表示成速度与 温度梯度的点积形式, 这个夹角就发挥了重要作用. 这 样的推导不可不谓雅致、简单、清新.

\section{3 应用广泛}

我们可从以下 5 个方面来说明场协同原理应用的 广泛性。

(1) FSP将现有的强化单向对流传热的机制统一 了起来 ${ }^{[11]}$. 文献中关于强化单相对流传热的机制有: 减薄热边界层厚度、增加流体中的扰动以及增加壁面 附近的速度梯度. 而数值模拟结果表明, 三者最终都是 改善了速度与温度梯度之间的协同性 ${ }^{[11]}$. 例如, 对于纵 

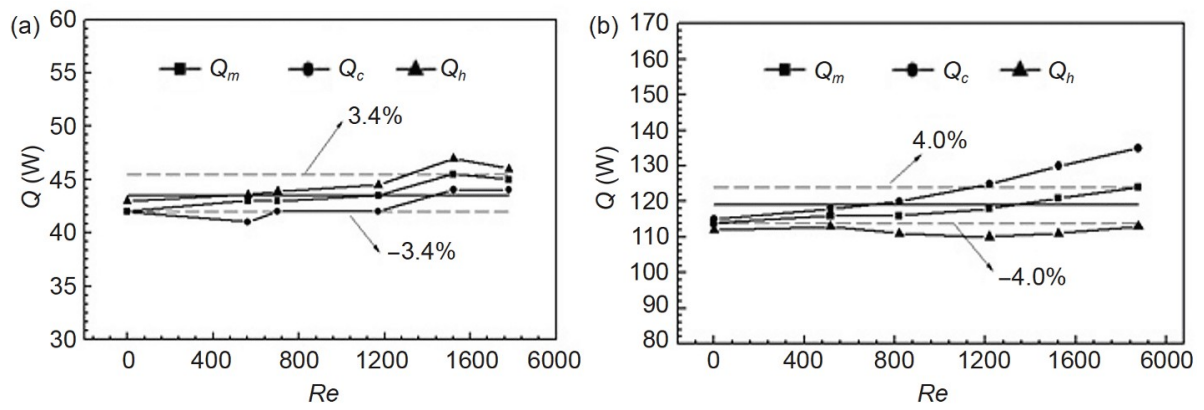

图 5 文献[15]的部分实验结果. (a) $\Delta T=10^{\circ} \mathrm{C}$; (b) $\Delta T=30^{\circ} \mathrm{C}$

Figure 5 Partial experimental results of ref. [15]. (a) $\Delta T=10^{\circ} \mathrm{C}$; (b) $\Delta T=30^{\circ} \mathrm{C}$.

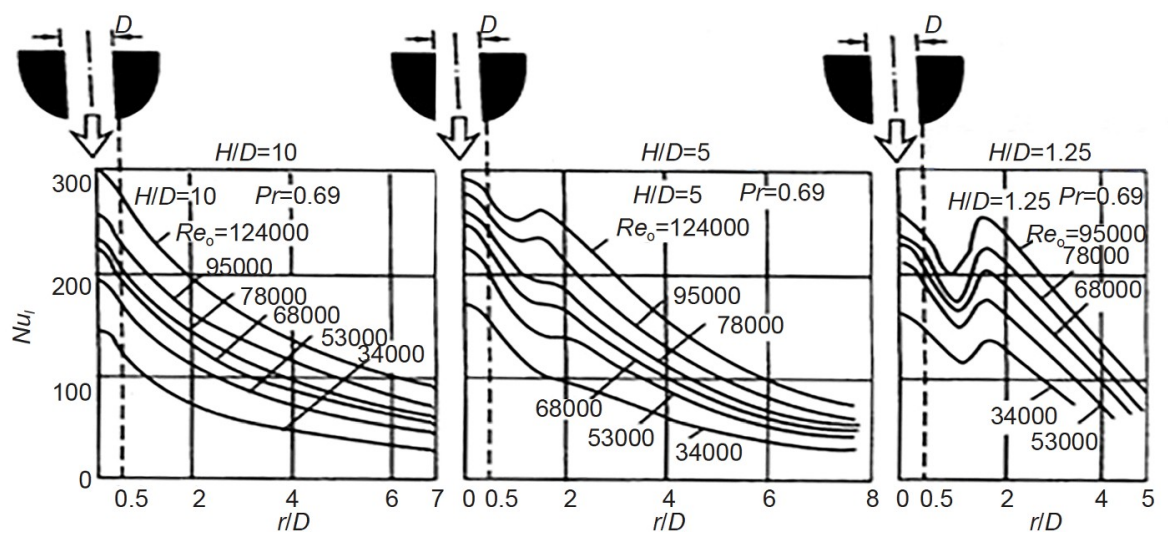

图 6 射流冲击对流传热局部对流传热系数随地点的变化 ${ }^{[19]}$

Figure 6 Local convective heat transfer coefficient varying with location in jet impingement [19].

向浴发生器, 文献中曾经提出了不少强化机制, 但文献 [17]的研究表明, 纵向浴发生器归根结底也还是改善 了速度与温度梯度之间的协同性.

(2) FSP既适用于不可压缩的抛物型流动、椭圆 型流动，也适用于可压缩流的双曲型流动. 上述场协 同的讨论都是对于不可压缩流动做出的. 文献[18]的 研究表明, 只要把温度梯度改为总焓梯度, 则FSP也适 合于可压缩流体的双曲型流动.

(3) FSP可以阐释许多现有的传热学研究结果的 物理机制. 冲击射流是一种高效的对流传热方式，特 别是在迟滞点处具有极高的对流传热系数 ${ }^{[19]}$ (参见图6 所示), 但其机理一直未能得到满意的说明. FSP对此给 出了满意的解释：在迟滞点处速度与流体的温度梯度 在一条直线上，是最理想的对流传热情形，因此具有 非常高的对流传热强度.

又例如，根据 $\mathrm{Gao}$ 等人 ${ }^{[20]}$ 的研究，图7中两种空气 太阳能集热器，平板式(图7(a))的效率很低，而无玻璃 散发式(图7(b))集热器效率较高. 通过数值模拟，文献 [20]的作者发现对于平板式集热器, 空气的流线几乎 与等温线平行(图8(a)), 意味着速度几乎与温度梯度垂 直; 而在散发式集热器中大部分地区流线几乎与等温 线垂直，就是该地区速度与温度梯度几乎同向(图8 (b)), 因此传热效率很高. 计算表明, 在同样的外界条 件下，平板式集热器的平均场协同角为 $88.3^{\circ}$, 而散发 式的仅 $42.7^{\circ}$.

（4）FSP可指导强化传热新技术的开发. FSP也为 开发强化传热技术指明了方向: 要设法减小协同角. 例 如, 清华大学的孟继安研究员据此思想开发了交叉扭 转椭圆管 ${ }^{[21]}$, 如图9所示. 作为一种独立的传热性能考 核, 我们根据清华大学提供的试验管, 进行了部分试验 研究, 验证了交叉扭转椭圆管的高效传热特性. 为查明 其机理, 特别进行了层流及湍流传热时的数值模拟, 部 分结果如图10所示 ${ }^{[22]}$. 由图10(a)和(b)可见, 无论是层 流还是湍流, 对于平直的椭圆截面管, 截面上流速与等 

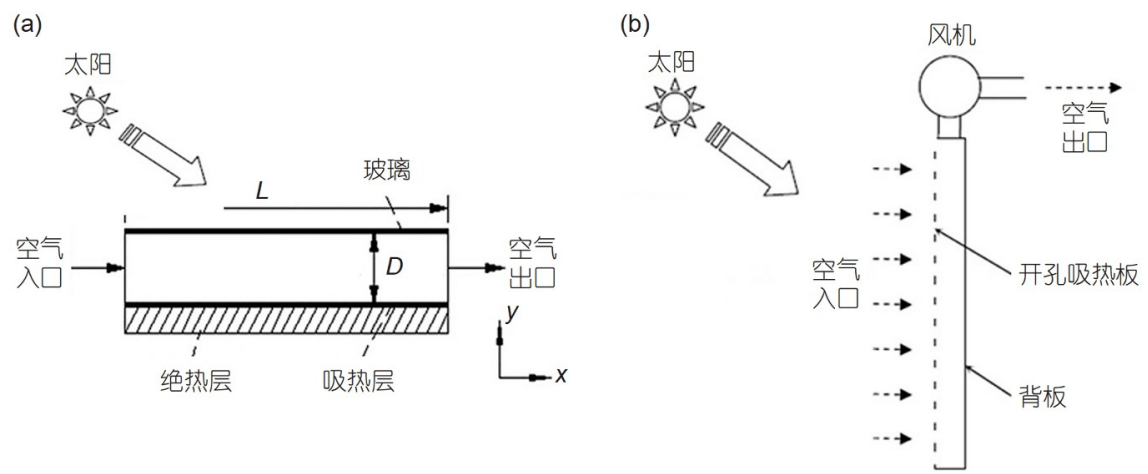

图 7 两种太阳能集热器. (a) 平板式集热器; (b) 无玻璃散发式集热器

Figure 7 Two kinds of solar collectors. (a) Flat plate collector; (b) unglazed transpired collector.

(a)
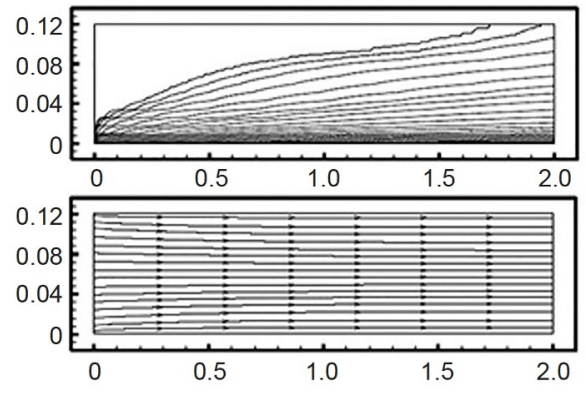

(b)

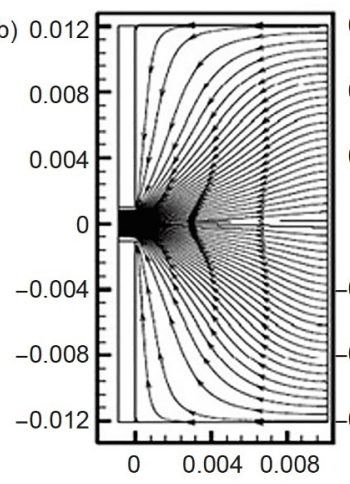

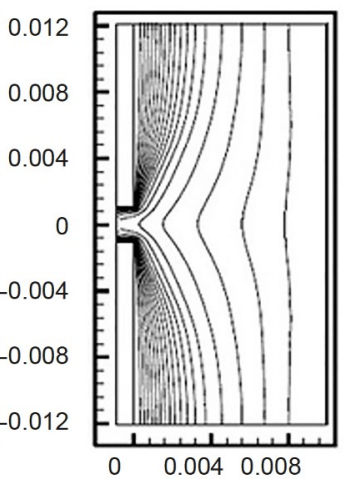

图 8 两种太阳能集热器中空气流线及等温线的分布 ${ }^{[20]}$. (a) 平板式集热器; (b) 无玻璃散发式集热器

Figure 8 Distribution of air flow lines and isotherms in two kinds of solar collectors [20]. (a) Flat plate collector; (b) unglazed transpired collector.

(a)

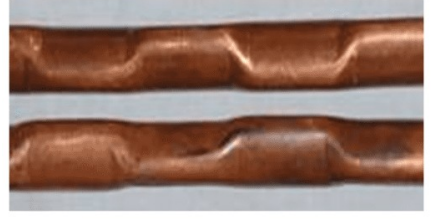

(b)

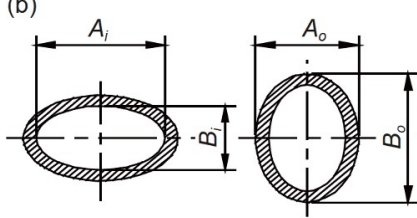

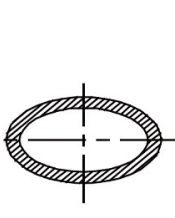

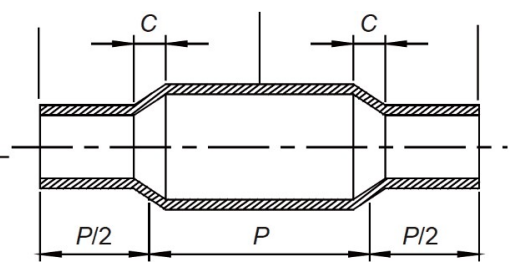

图 9 (网络版彩图) (a) 交叉扭转椭圆管; (b) 截面图

Figure 9 (Color online) (a) Alternating elliptical axis tube; (b) sections.

温线(图中连续的曲线)几乎平行，而对于交叉扭转椭 圆管在截面的长短轴两端有 4 个局部区域，那里的流 速与等温线几乎垂直, 也就是流速与温度梯度几乎在 一条线上, 局部协同性很好, 使得传热得以强化. 图10 (c)显示了椭圆截面直管的平均协同角大于交叉扭转 椭圆管. 虽然对所研究的情形, 差别只有 $1^{\circ}$ 左右, 但在 这样大的角度范围内, $1^{\circ}$ 的差别就会使余弦有百分之 几十、甚至成倍的差别. 此外, 西安交通大学新研发
出的前疏后密开缝翅片 ${ }^{[23,24]}$, 利用新型的纵向浴结构 来强化传热等 ${ }^{[25,26]}$, 都是在FSP指导下实现的. 更多的 例子可参见文献[27].

(5) FSP的基本思想有发展空间, 并可推广应用于 其他领域. 作者及同事拓展FSP的基本思想, 并应用于 其他领域, 特别是脉管制冷机. 大量研究 ${ }^{[28]}$ 发现, 凡是 物理过程的结果取决于两个矢量的点积的, 则减小矢 量的夹角均可强化该物理过程, 如表1所示. 
由表1可见，对于脉管制冷机，其制冷量的大小与 气体速度与压力矢量之间的协同(相位差)密切相关, 脉管制冷机从第一代到第三代的发展(图11)，分析其 不断改善的根本原因, 主要是在改善这个协同度上取 得了进展. 对于同一种脉管制冷机，其几何结构对性 能的影响也主要体现在速度与压力的协同或相位差 上. 例如, 由西安交通大学朱绍伟、吴沛宜等发明的双 向进气脉管制冷机的制冷性能，在文献[28]模拟的条 件下，其长径比 $(L / D)$ 与制冷量及相位差的关系显示在 图12中. 由图12可见，当速度与压力达到最好的协同 (最小的相位差)时, 脉管的冷却能力最大

\section{4 场协同原理的自然性}

在积分后的边界层能量方程中，将对流项用速度 与温度梯度代替后就能很自然地引出了 FSP的基本思 想：增加速度与温度梯度夹角余弦的绝对值可以增加 对流传热量(在其他条件相同下)，因而强化对流传热， 其中没有引入其他任何复杂的变换. 这个速度与温度 梯度夹角的重要作用，在边界层能量方程中隐藏了大
半个世纪后才被发现.

\section{5 场协同原理的对称性}

对称性是人们日常生活中常见的特性，例如雪花 是对称的(图13(a)), 人的脸基本是对称的(图13(b)). 对 称性给人带来的美感，在与非对称的情形作对比后就 能更显著地体现出来(图13(c)).

那么什么是科学原理上的对称性呢? 按照文献 [6]: 任何运算(操作)导致某些特性不变时, 称该特性对 此运算(操作)具有对称性. 据此, FSP的对称性可从图14 得到说明，对于速度与温度梯度垂直的情形(图14(a)), 改变速度的方向，不改变传热量(0); 而对于速度与温 度梯度平行的情形(图14(b)), 改变速度的方向也不改 变传热量, 即流体从被加热变为被冷却，但对流传热 量不变.

\section{6 场协同原理的比拟性}

场协同原理的比拟性可用图15来表达，该图的意 义正体现了FSP的通俗易懂的比拟性，即流体速度和
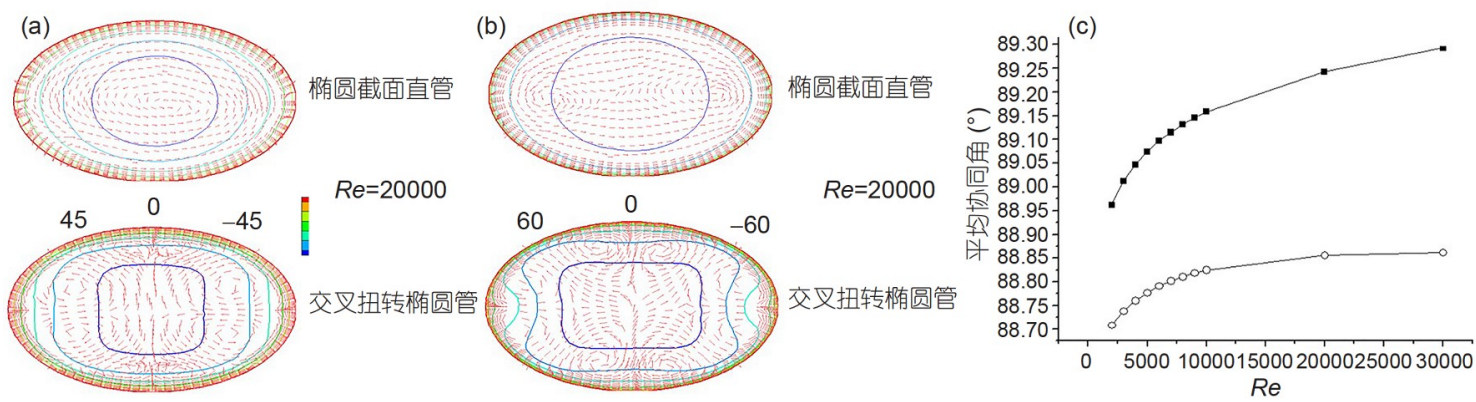

图 10 (网络版彩图)交叉扭转椭圆管传热特性数值模拟结果. (a) $R e=2000$; (b) $R e=20000$; (c) 平均协同角

Figure 10 (Color online) Numerical simulation results of heat transfer characteristics of alternating elliptical axis tube. (a) $R e=2000$; (b) $R e=20000$; (c) average synergy angle.

表 1 物理过程取决于两个矢量点积的例子

Table 1 Examples showing the physical processes which depend on the dot product of two vectors

\begin{tabular}{ccccc}
\hline 物理现象 & 广义力 & 广义流 & 广义能 & 所传递的量 \\
\hline 对流传热 & 温度梯度 $\nabla T$ & 流体速度 & $q \infty \nabla T \cdot \mathbf{u}$ \\
力做功 & 力 $\mathbf{F}$ & 热量 & $w \infty \mathbf{F} \cdot \Delta \mathbf{x}$ \\
极化 & 位移 $\Delta \mathbf{x}$ & 功 & $w \infty \mathbf{E} \cdot \Delta \mathbf{P}$ \\
磁化 & 外磁场强度 $\mathbf{E}$ & 极化强度 $\Delta \mathbf{P}$ & 极化电功 & $w \infty \mathbf{H} \cdot \Delta \mathbf{M}$ \\
脉管制冷机 & 压力场 & 磁化强度 $\Delta \mathbf{M}$ & 功 & $q \infty \mathbf{p} \cdot \mathbf{u}$ \\
斯特林机 & 压力场 & 流体速度 & 能力 & $q \infty \mathbf{p} \cdot \mathbf{u}$ \\
\hline
\end{tabular}




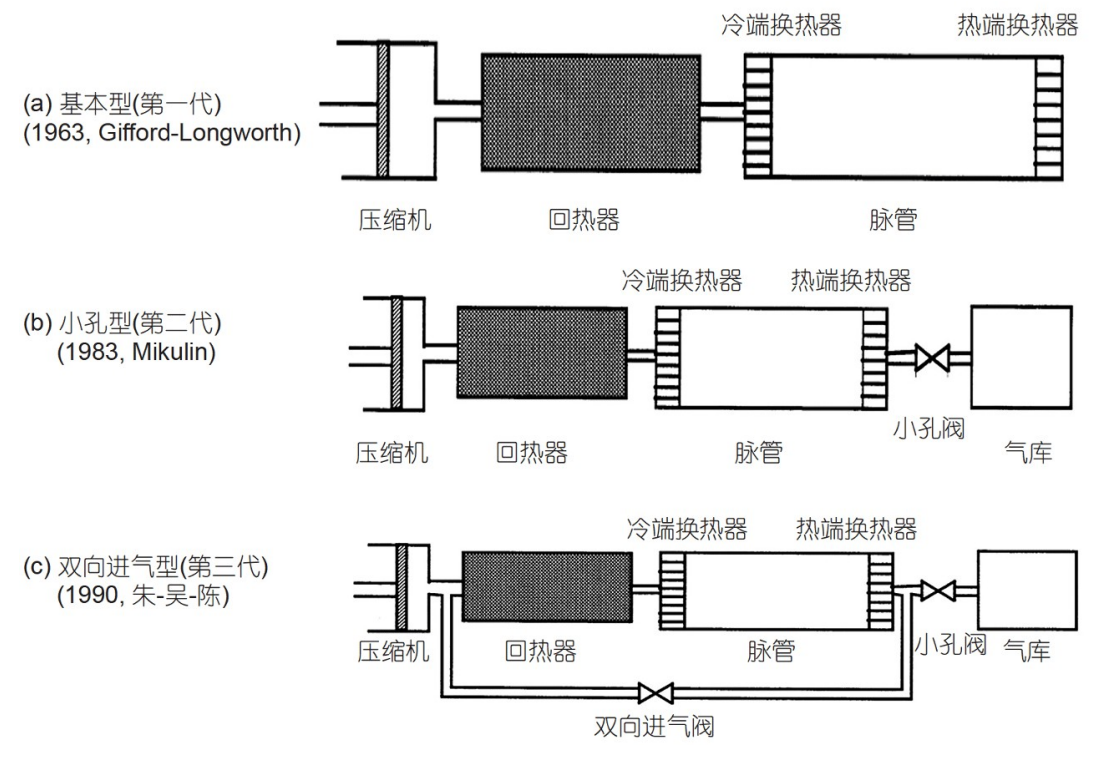

图 11 三代脉管制冷机示意图 ${ }^{[28]}$

Figure 11 Schematic diagram of the three-generation pulse tube refrigerators [28].
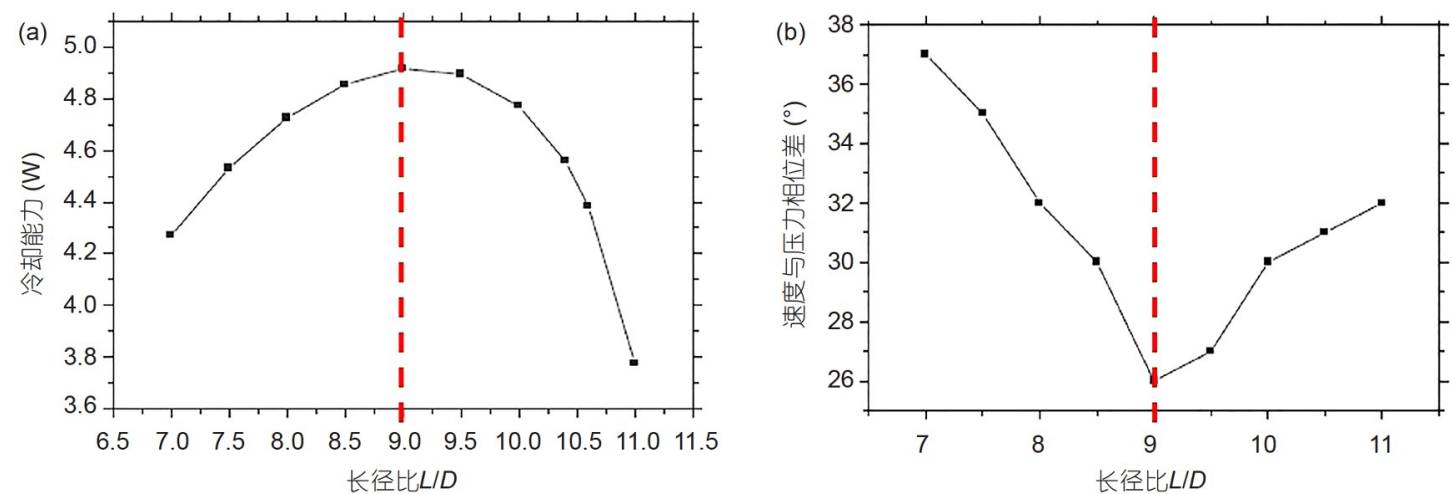

图 12 (网络版彩图)长径比对双向进气脉管制冷机性能的影响 ${ }^{[28]}$. (a) 最大制冷能力; (b) 最佳长径比

Figure 12 (Color online) Influence of length-diameter ratio on the performance of bidirectional intake pulse tube refrigerator [28]. (a) Maximum cooling capacity; (b) optimal aspect ratio.

(a)

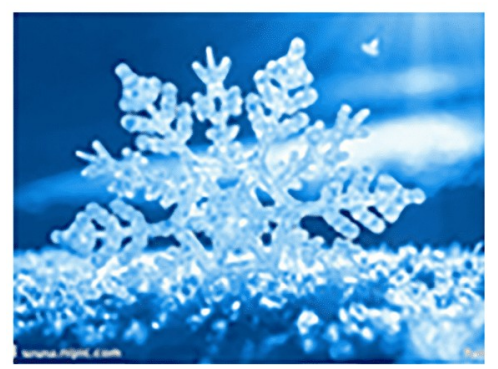

(b)

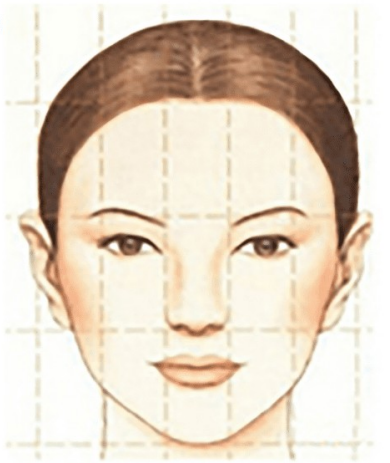

(c)

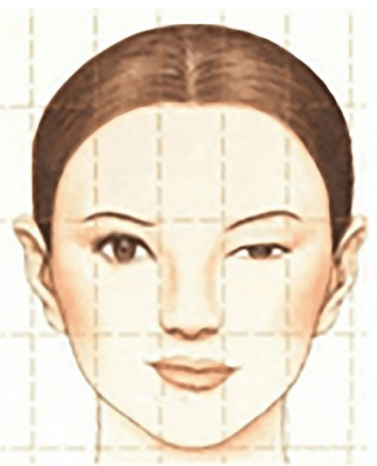

图 13 (网络版彩图)对称性给人以美感. (a) 雪花; (b) 基本对称的脸; (c) 不对称的脸

Figure 13 (Color online) Symmetry gives a sense of beauty. (a) Snow; (b) symmetrical face; (c) asymmetrical face. 

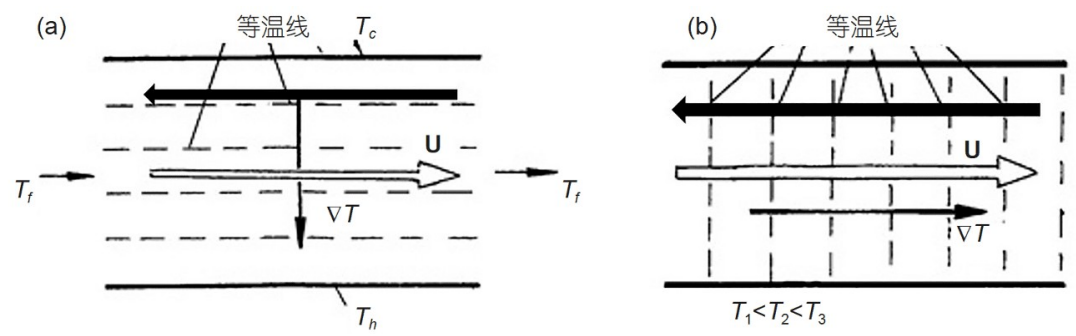

图 14 FSP的对称性. (a) 速度与温度梯度垂直; (b) 速度与温度梯度平行

Figure 14 Symmetry of FSP. (a) The velocity is perpendicular to the temperature gradient; (b) the velocity is parallel to the temperature gradient.
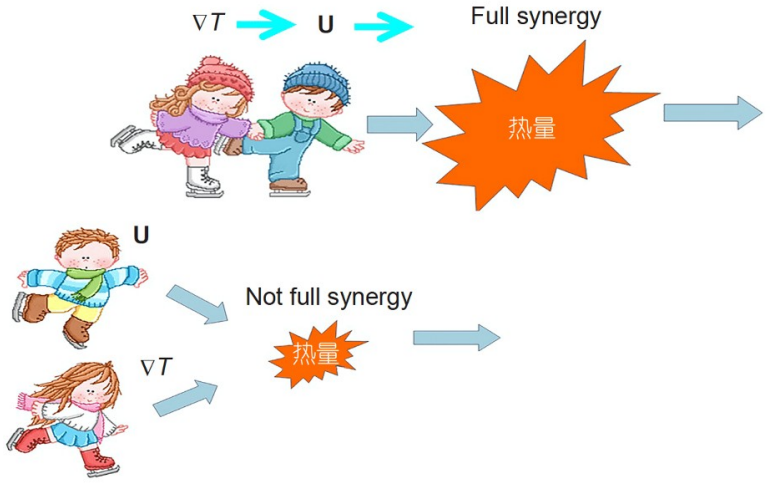

图 15 (网络版彩图) FSP的比拟性

Figure 15 (Color online) Analogy of FSP.
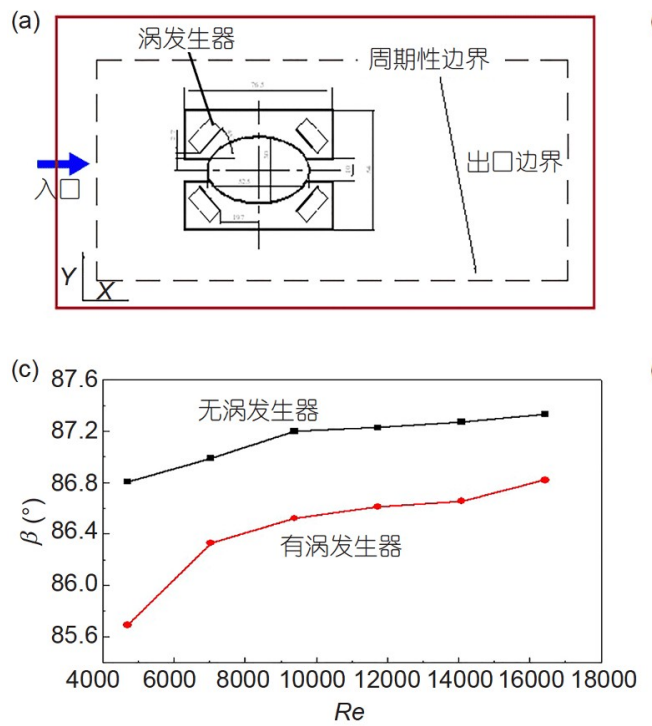

图 16 (网络版彩图)说明场协同原理与熄理论一致性的例子 ${ }^{[30]}$. (a) 带纵向浴的 $\mathrm{H}$ 翅片; (b) $N u$ 与 $R e$ 的关系; (c) 协同角与 $R e$ 的 关系; (d) 炇耗散与 Re的关系

Figure 16 (Color online) The example which illustrates the consistency between field synergy principle and entransy theory [30]. (a) $\mathrm{H}$ fins with longitudinal vortexes; (b) relation between $\mathrm{Nu}$ and $R e$; (c) relation between synergy angle and $R e$; (d) relation between entransy dissipation and $R e$.
温度梯度都可以比拟为力, 它们推动对流换热的传热 量, 由于它们都是矢量, 其合力必与它们的夹角有关.

\section{3 场协同原理与沵理论的关系}

关于场协同原理与积理论 ${ }^{[29]}$ 的关系，本文作者做 过专题研究 ${ }^{[30]}$, 我们对6种强化传热的几何结构, 在层 流与湍流、给定壁温及给定热流等多种条件的强化特 性与基准表面进行了比较, 分别用FSP及䍉理论进行了 分析, 发现其结果完全一致, 说明了两者之间有内在的 (图16(a)) 及没有纵向浴的 $\mathrm{H}$ 型翅片 (均为给定壁温)在

(b)
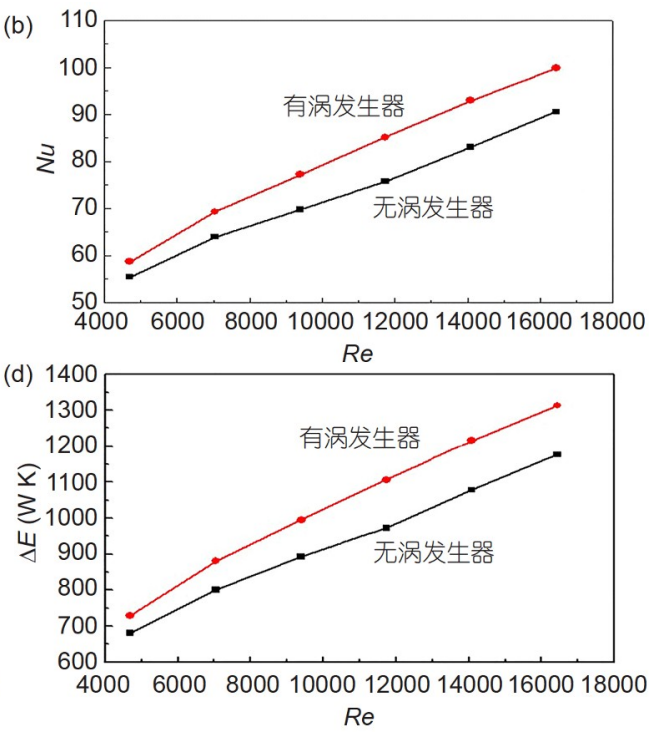
一致性. 例如, 图16中给出了对于带纵向浴的 $\mathrm{H}$ 型翅片 


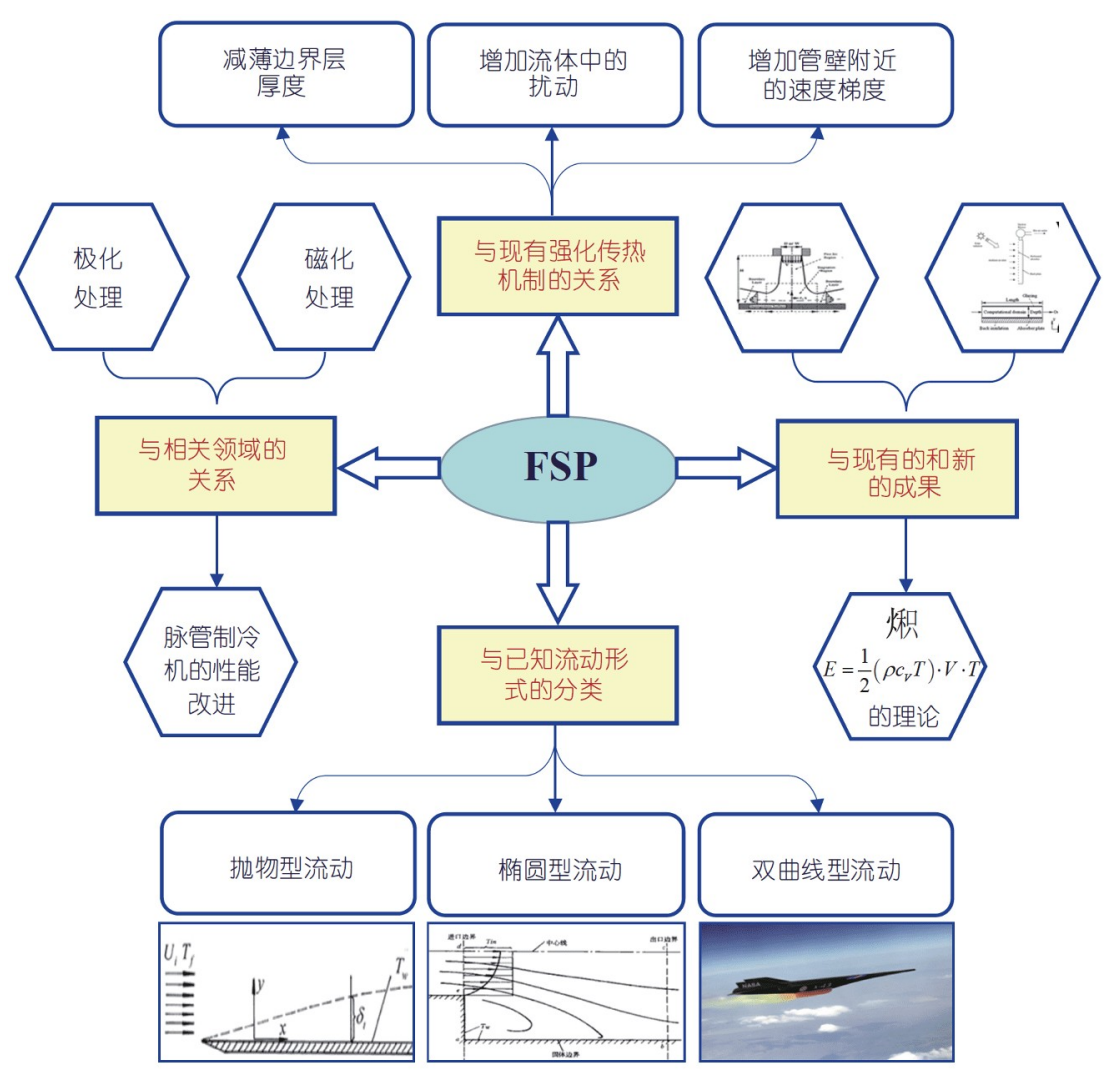

图 17 (网络版彩图)场协同原理应用的广泛性

Figure 17 (Color online) Wide application of field synergy principle.

湍流情况下的模拟结果. 由图可见, 带纵向涡的H翅片 传热优于无纵向浴的翅片(图16(b)), 其协同角明显小 于无纵向浴的翅片(图16(c)), 而其炇耗散则大于无纵 向浴的翅片(图16(d)). 这些结果完全协调一致.

至此，我们可以对场协同原理应用给出如图17所 示的描述，该图可以充分表达场协同原理科学之美的 重要特性之一——应用的广泛性.

\section{4 结论}

场协同原理是具有科学之美各种特性的科学理 论, 其概念清晰、推导优雅、应用广泛, 且具有自 然性、对称性和比拟性; 同时, 其与炽理论相互论 证、相辅相成, 对传热学的发展具有重要完善和推动 作用.

致谢 感谢作者的研究生们在校期间为执行本文的研究项目付出的努力; 感谢西安交通大学航天航空学院俞茂宏教授 给作者分享他2004年在国际强度理论大会的总结会上所做的关于统一强度理论科学之美的报告，对本文的写作具有重 要的帮助; 感谢清华大学过增元院士同意本文使用他设计的表述FSP比拟性的图案(本文图15); 感谢西安交通大学热流 科学与工程教育部重点实验室的郑春宇老师在文献和资料的收集方面所提供的帮助.

\section{参考文献}

1 Zhu G Q. Zhu Guangqian Talks About Beauty (in Chinese). Shanghai: East China Normal University Press, 2012 [朱光潜. 朱光潜谈美. 上海: 华 东师范大学出版社, 2012] 
2 Yu M H. New system of Strength Theory: Theory, Development and Application (in Chinese). 2nd ed. Xi'an: Xi'an Jiaotong University Press, 2011 [俞茂宏. 强度理论新体系: 理论、发展和应用. 第2版. 西安: 西安交通大学出版社, 2011]

3 Tian G. Inside and outside of mathematics (in Chinese). https://baijiahao.baidu.com/s?id=1668920502802121995 [田刚. 数学内外. https:// baijiahao.baidu.com/s?id=1668920502802121995]

4 Peng S G. Theory, methods and meaning of nonlinear expectation theory (in Chinese). Sci Sin-Math, 2017, 47: 1223-1254 [彭实戈. 非线性期望 的理论、方法及意义. 中国科学: 数学, 2017, 47: 1223-1254]

5 https://mp.weixin.qq.com/s/n1aqBPkEMQN5GHnv5fleDQ

6 Tzanakis C. The quest of beauty in research and teaching of mathematics and physics: A historical approach. Nonlinear Anal-Theory Methods Appl, 1997, 30: 2079-2105

7 Kosso P. Symmetry arguments in physics. Studies History Philosophy Sci Part A, 1999, 30: 479-492

8 Guo Z Y, Li D Y, Wang B X. A novel concept for convective heat transfer enhancement. Int J Heat Mass Transfer, 1998, 41: 2221-2225

9 Neufeldt V, Guralnik D B. Webster's New World Dictionary of American English. 3rd ed. New York: Prentice Hall, 1994

10 Tao W Q, Guo Z Y, Wang B X. Field synergy principle for enhancing convective heat transfer-Its extension and numerical verifications. Int J Heat Mass Transfer, 2020, 45: 3849-3856

11 Tao W Q, He Y L, Wang Q W, et al. A unified analysis on enhancing single phase convective heat transfer with field synergy principle. Int J Heat Mass Transfer, 2020, 45: 4871-4879

12 Zeng M, Tao W Q. Numerical verification of the field synergy principle for turbulent flow. J Enhanc Heat Transf, 2004, 11: 451-457

13 Yang S M, Tao W Q. Heat Transfer (in Chinese). 4th ed. Beijing: High Education Press, 2006 [杨世铭, 陶文铨. 传热学. 第4版. 北京: 高等教育 出版社, 2006]

14 Zhao T S, Song Y J. Forced convection in a porous medium heated by a permeable wall perpendicular to flow direction: Analyses and measurements. Int J Heat Mass Transfer, 2001, 44: 1031-1037

15 Ma L D, Li Z Y, Tao W Q. Experimental verification of the field synergy principle. Int Commun Heat Mass Transfer, 2007, 34: 269-276

16 Layton E T, Lienhard J H. History of Heat Transfer. New York: ASME, 1988

17 Wu J M, Tao W Q. Numerical study on laminar convection heat transfer in a rectangular channel with longitudinal vortex generator. Part A: Verification of field synergy principle. Int J Heat Mass Transfer, 2008, 51: 1179-1191

18 Li X, He Y L, Tao W Q. Analysis and extension of field synergy principle (FSP) for compressible boundary-layer heat transfer. Int J Heat Mass Transfer, 2015, 84: 1061-1069

19 Martin H. Heat and mass transfer between impinging gas jets and solid surfaces. Adv Heat Transfer, 1977, 13: 1-60

20 Gao L, Bai H, Wu X. Numerical analysis of heat transfer in unglazed transpired collectors based on field synergy principle. Sol Energy, 2013, 95: 336-344

21 Meng J A. Enhanced heat transfer technology of longitudinal vortices based on field-coordination principle and its application (in Chinese). Dissertation for Doctoral Degree. Beijing: Tsinghua University, 2003 [孟继安. 基于场协同理论的纵向浴强化换热技术及其应用. 博士学位 论文. 北京: 清华大学, 2003]

22 Li B, Feng B, He Y L, et al. Experimental study on friction factor and numerical simulation on flow and heat transfer in an alternating elliptical axis tube. Appl Thermal Eng, 2006, 26: 2336-2344

23 Cheng Y P, Qu Z G, Tao W Q, et al. Numerical design of efficient slotted fin surface based on the field synergy principle. Numer Heat Transf-Part A, 2004, 45: 517-538

24 Qu Z G, Tao W Q, He Y L. Three-dimensional numerical simulation on laminar heat transfer and fluid flow characteristics of strip fin surface with X-arrangement of strips. J Heat Transfer, 2004, 126: 697-707

25 Tian L T, He Y L, Lei Y G, et al. Numerical study of fluid flow and heat transfer in a flat-plate channel with longitudinal vortex generators by applying field synergy principle analysis. Int Commun Heat Mass Transfer, 2009, 36: 111-120

26 Li M J, Zhou W J, Zhang J F, et al. Heat transfer and pressure performance of a plain fin with radiantly arranged winglets around each tube in finand-tube heat transfer surface. Int J Heat Mass Transfer, 2014, 70: 734-744

27 He Y L, Tao W Q. Convective heat transfer enhancement: Mechanism, techniques and performance evaluation. Adv Heat Transf, 2014, 46: 87186

28 He Y L, Wu M, Tao W Q, et al. Improvement of the thermal performance of pulse tube refrigerator by using a general principle for enhancing 
energy transport and conversion processes. Appl Thermal Eng, 2004, 24: 79-93

29 Guo Z Y, Zhu H Y, Liang X G. Entransy-A physical quantity describing heat transfer ability. Int J Heat Mass Transfer, 2007, 50: 2545-2556

30 He Y L, Tao W Q. Numerical studies on the inherent interrelationship between field synergy principle and entransy dissipation extreme principle for enhancing convective heat transfer. Int J Heat Mass Transfer, 2014, 74: 196-205

\title{
Field synergy principle: Its scientific beauty and relationship with entransy theory
}

\author{
TAO WenQuan ${ }^{1,2}$ \& HE YaLing ${ }^{1,2}$ \\ ${ }^{1}$ Key Laboratory of Thermo-Fluid Science and Engineering of Ministry of Education, Xi'an Jiaotong University, Xi'an 710049, China; \\ ${ }^{2}$ Int'l Joint Research Laboratory of Thermal Science and Engineering, Xi'an Jiaotong University, Xi'an 710049, China
}

From the six aspects of the beauty of science, this paper analyzes and discusses the beauty of the science of the field synergy principle (FSP) proposed by Guo. Its clear concept, elegant derivation, and wide application are analyzed. Its naturality, symmetry, and analogy are discussed. Finally, the inherent relationship between FSP and entransy theory is revealed. The paper shows that FSP and entransy theory prove and complement each other, and thus both play important roles in improving and promoting the development of knowledge of heat transfer.

beauty of science, field synergy principle, entransy theory

doi: $10.1360 /$ SST-2021-0138 